\title{
The Design of a Class of Perfect Reconstruction Two-Channel FIR Linear-Phase Filterbanks and Wavelets Bases Using Semidefinite Programming
}

\author{
S. C. Chan, Carson K. S. Pun, and K. L. Ho
}

\begin{abstract}
This paper proposes a new method for designing a class of two-channel perfect reconstruction (PR) linear-phase FIR filterbanks (FBs) and wavelets previously proposed by Phoong et al. By expressing the given $K$-regularity constraints as a set of linear equality constraints in the design variables, the design problem using the minimax error criterion can be solved using semidefinite programming (SDP). Design examples show that the proposed method is very effective and it yields equiripple stopband response while satisfying the given $K$-regularity condition.
\end{abstract}

Index Terms-Filterbanks, perfect reconstruction, regularity, semidefinite programming, wavelets.

\section{INTRODUCTION}

$\mathbf{P}$ ERFECT RECONSTRUCTION (PR) multirate filterbanks (FBs) have important applications in signal analysis, signal coding and the design of wavelet bases. A number of techniques for designing linear-phase and low-delay two-channel PR FIR FBs are now available [1]-[7]. The structurally PR two-channel FIR FBs proposed in [1] are particularly attractive because the PR property is structurally imposed and their design and implementation complexities are very low. The FBs are parameterized by two functions $\beta(z)$ and $\alpha(z)$, and some delay parameters. In [1], these two functions were chosen to be identical to realize FIR and IIR FBs with very good frequency characteristics [1]. The construction of wavelet bases from these structurally PR FBs having the maximally possible $K$-regularity condition was also considered. Later in [2], the construction of low-delay FBs and wavelet bases satisfying the $K$-regularity condition up to one zero-order moment was also studied. The incorporation of $K$-regularity with higher order, however, was not addressed due to the difficulty in solving the constrained optimization problem. The design of paraunitary two-channel FIR FBs [the conjugate quadrature filters $(\mathrm{CQF})]$ with prescribed number of $K$-regularity was previously studied in [5]. The wavelets FBs so obtained are in general not linear-phase. Recently, Tay [6] showed that it is possible to incorporate the $K$-regularity condition (or equivalently a certain number of zeros in the lowpass analysis filter and highpass analysis filter at $\omega=\pi$ and $\omega=0$, respectively) for the linear-phase FBs in [1] using the Bernstein polynomial. Since the error function is a linear function of the coefficients in the Bernstein polynomial, it can be solved as a simple quadratic programming problem if the least squares error

Manuscript received August 15, 2002; revised December 5, 2002. The associate editor coordinating the review of this manuscript and approving it for publication was Prof. Yu-Hen Hu.

The authors are with the Department of Electrical and Electronic Engineering, The University of Hong Kong, Hong Kong (e-mail: scchan@eee.hku.hk; kspun@eee.hku.hk; klho@eee.hku.hk).

Digital Object Identifier 10.1109/LSP.2003.821688 criterion is used. The Genetic Algorithm (GA) was also proposed for designing such FBs and wavelets with higher order moments and sum-of-powers-of-two (SOPOT) coefficients [3].

In this paper, we extend the work in [2] to the case of higher order $K$-regularity [5]. This will enable a larger class of wavelet FBs with different smoothness and system delay to be constructed. More precisely, by expressing the given $K$-regularity constraints as a set of linear equality constraints in the design variables, the design problem with the minimax error criterion can be solved using semidefinite programming (SDP) [8]-[11]. Thanks to the interior-point method [11], SDP can be solved efficiently in polynomial time. Design results show that the proposed design method yields PR linear-phase FBs with equiripple stopband characteristics, while satisfying the required $K$-regularity condition. The paper is organized as follows. In Section II, the proposed SDP design method will be described. Several design examples will then be given in Section III. Finally conclusions are drawn in Section IV.

\section{Proposed Design Method}

Fig. 1 shows the structurally PR FBs [1], [2] that we are going to design. The FBs are parameterized by the subfilter pairs $\beta(z)$ and $\alpha(z)$ and the delay parameters $N$ and $M$. The FBs are PR for arbitrary choice of filter pairs $\beta(z)$ and $\alpha(z)$, which can be chosen as linear-phase FIR or all-pass functions to realize FIR and IIR FBs with very low design and implementation complexities. In [1], the case of using identical $\alpha(z)$ and $\beta(z)$ with delay parameter $M=2 N-1$ was studied so as to obtain linear-phase FIR or passband linear-phase all-pass based IIR FBs. Later in [2], $\alpha(z)$ and $\beta(z)$ are generalized to include general FIR functions. For simplicity, we shall consider the case where $\alpha(z)$ and $\beta(z)$ are linear-phase functions. From Fig. 1, we obtain the frequency responses of the analysis filters as follows:

$$
\begin{aligned}
& H_{0}\left(e^{j \omega}\right)=\frac{1}{2}\left(e^{-j 2 N \omega}+e^{-j \omega} \beta\left(e^{j 2 \omega}\right)\right) \\
& H_{1}\left(e^{j \omega}\right)=-\alpha\left(e^{j 2 \omega}\right) H_{0}\left(e^{j \omega}\right)+e^{j(-2 M-1) \omega} .
\end{aligned}
$$

Let $L_{\beta}$ and $L_{\alpha}$ be respectively the lengths of $\beta\left(e^{j \omega}\right)$ and $\alpha\left(e^{j \omega}\right)$. In [2], the desired responses of $\beta\left(e^{j \omega}\right)$ and $\alpha\left(e^{j \omega}\right)$ are studied. It can be shown that the ideal responses of $\beta\left(e^{j \omega}\right)$ and $\alpha\left(e^{j \omega}\right)$ are given respectively by

$$
\begin{aligned}
& \beta_{d}\left(e^{j \omega}\right)=e^{j\left(-N+\frac{1}{2}\right) \omega} \\
& \alpha_{d}\left(e^{j \omega}\right)=\frac{e^{j\left(-M-\frac{1}{2}\right) \omega}}{H_{0}\left(e^{\frac{j \omega}{2}}\right)}, \quad \omega \in[-\pi, \pi] .
\end{aligned}
$$

From (2), we can see that $\beta\left(e^{j 2 \omega}\right)$ approximates $e^{-j(2 N-1) \omega}$ for $0<\omega<\pi / 2$. Due to the periodic nature of $\beta\left(e^{j 2 \omega}\right)$, for 

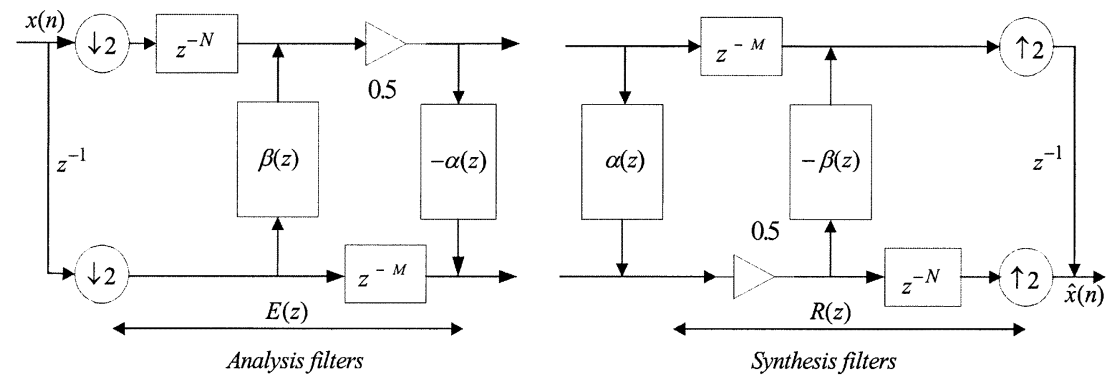

Fig. 1. Two-channel structurally PR FBs.

$\pi / 2<\omega<\pi, \beta\left(e^{j 2 \omega}\right)$ becomes anti-phase with $e^{-j(2 N-1) \omega}$ and therefore cancel each other. Hence, $H_{0}\left(e^{j \omega}\right)$ approximates $e^{-j(2 N-1) \omega}$ in the passband and becomes zero for $\pi / 2<\omega<$ $\pi$. As for $\alpha\left(e^{j 2 \omega}\right)$, the product $H_{0}\left(e^{j \omega}\right) \alpha\left(e^{j 2 \omega}\right)$ will approximate $e^{j(-2 M-1) \omega}$ for $0<\omega<\pi / 2$. Therefore, $H_{1}\left(e^{j \omega}\right)$ will nearly be zero in the stopband, $0<\omega<\pi / 2$. Since $H_{0}\left(e^{j \omega}\right)$ is approximately zero in $\pi / 2<\omega<\pi$ and $\alpha\left(e^{j 2 \omega}\right)$ has a magnitude approximately less than one, $H_{1}\left(e^{j \omega}\right)$ will be close to $e^{j(-2 M-1) \omega}$ in the passband.

To construct wavelet FBs, the analysis filters $H_{0}\left(e^{j \omega}\right)$ and $H_{1}\left(e^{j \omega}\right)$ should possess at least one zero at $\omega=\pi$ and $\omega=0$, respectively. A higher order of $K$-regularity can be obtained by imposing $K_{0}$ and $K_{1}$ zeros at $\omega=\pi$ and $\omega=0$ for $H_{0}\left(e^{j \omega}\right)$ and $H_{1}\left(e^{j \omega}\right)$, respectively (with $K_{0} \geq K_{1}>1$ ). This is equivalent to the following:

$$
\left[\frac{d^{k_{0}}}{d \omega^{k_{0}}} H_{0}\left(e^{j \omega}\right)\right]_{\omega=\pi}=\left[\frac{d^{k_{1}}}{d \omega^{k_{1}}} H_{1}\left(e^{j \omega}\right)\right]_{\omega=0}=0
$$

for $k_{0}=0, \cdots, K_{0}-1$ and $k_{1}=0, \cdots, K_{1}-1$. After performing the differentiation, it can be shown that the regularity constraints in (3) can be expressed in terms of the coefficients of $\beta(z)$ and $\alpha(z)$ as shown at the bottom of page for $k_{0}=$ $0, \cdots, K_{0}-1$ and $k_{1}=0, \cdots, K_{1}-1$. $\lceil x\rceil$ denotes the integer which is just greater than or equal to $x$. It can be seen that (4a) is a set of linear equality constraints in $\boldsymbol{\beta}$ and $\boldsymbol{\alpha}$, the vectors containing the coefficients of $\beta(z)$ and $\alpha(z)$, respectively. Equation (4a) can also be written more compactly in matrix form as

$$
\boldsymbol{B} \cdot \boldsymbol{\beta}=\boldsymbol{c}_{\beta, K_{0}} \quad \boldsymbol{A} \cdot \boldsymbol{\alpha}=\boldsymbol{c}_{\alpha, K_{1}}
$$

for some matrices $\boldsymbol{B}$ and $\boldsymbol{A}$, and vectors $\boldsymbol{c}_{\beta, K_{0}}$ and $\boldsymbol{c}_{\alpha, K_{1}}$, whose entries are defined in (4a). First of all, let us consider the design of $\beta(z)$. To minimize the maximum ripple of the approximation error is equivalent to

$$
\min _{\beta}\left|\boldsymbol{\beta}^{T} \cdot \boldsymbol{e}-\beta_{d}\left(e^{j \omega}\right)\right|^{2}, \quad \text { for } \omega \in\left[0,2 \omega_{\beta p}\right]
$$

where

$$
e=\left[\begin{array}{llll}
1 & e^{-j \omega} & \cdots & e^{-j\left(L_{\beta}-1\right) \omega}
\end{array}\right]
$$

To solve (5) using SDP, we densely discretize $\omega$ over the band of interest $\omega \in\left[0,2 \omega_{\beta p}\right]$ into a set of frequencies points $\omega_{k}$ 's, $k=1, \cdots K$. Hence, the optimization problem in (5) is approximated as

$$
\begin{aligned}
& \min \delta \\
& \eta_{R, \beta}^{2}\left(\omega_{k}\right)+\eta_{I, \beta}^{2}\left(\omega_{k}\right) \leq \delta, \quad k=1, \cdots K
\end{aligned}
$$

where

$$
\begin{aligned}
\eta_{I, \beta}(\omega) & =\boldsymbol{\beta}^{T} \cdot \boldsymbol{s}+\boldsymbol{I} \boldsymbol{m}\left\{\beta_{d}\left(e^{j \omega}\right)\right\} \\
\eta_{R, \beta}(\omega) & =\boldsymbol{\beta}^{T} \cdot \boldsymbol{c}-\boldsymbol{R} \boldsymbol{e}\left\{\beta_{d}\left(e^{j \omega}\right)\right\} \\
\boldsymbol{c} & =\left[\begin{array}{cc}
\cos (0 \cdot \omega) & \cos (1 \cdot \omega) \\
\cdots & \cos \left(\left(L_{\beta}-1\right) \cdot \omega\right)
\end{array}\right] \\
\boldsymbol{s} & =\left[\begin{array}{cc}
\sin (0 \cdot \omega) & \sin (1 \cdot \omega) \\
\cdots & \sin \left(\left(L_{\beta}-1\right) \cdot \omega\right)
\end{array}\right]
\end{aligned}
$$

where $\operatorname{Re}\{x\}$ and $\boldsymbol{I} \boldsymbol{m}\{x\}$ denote the real and imaginary parts of $x$ respectively. Using Schur complement [8]-[10], it can be shown that [8] (6a) is equivalent to

$\min \delta$

$$
F_{k}(\boldsymbol{h}) \geq 0, \quad k=1, \cdots K \text {. }
$$

where $F_{k}(\boldsymbol{h})=\left(\begin{array}{ccc}\delta & \eta_{R, \beta}\left(\omega_{k}\right) & \eta_{I, \beta}\left(\omega_{k}\right) \\ \eta_{R, \beta}\left(\omega_{k}\right) & 1 & 0 \\ \eta_{I, \beta}\left(\omega_{k}\right) & 0 & 1\end{array}\right)$. Since $F_{k}(\boldsymbol{h})$ is affine in $\boldsymbol{\beta}$, it is equivalent to a set of linear matrix inequalities (LMI) [9]. Define the augmented variable $\boldsymbol{x}^{T}=$ $\left[\begin{array}{ll}\delta & \boldsymbol{\beta}^{T}\end{array}\right]$. The optimization problem in (6c) can be cast into the following standard LMI or SDP optimization problem:

$$
\begin{aligned}
& \min \boldsymbol{c}^{T} \cdot \boldsymbol{x} \\
& \boldsymbol{F}(\boldsymbol{x}) \geq 0
\end{aligned}
$$

where $\boldsymbol{F}(\boldsymbol{x})=\operatorname{diag}\left(F_{1}(\boldsymbol{x}), F_{2}(\boldsymbol{x}), \cdots F_{K}(\boldsymbol{x})\right)$. In order to simultaneously solve the SDP problem (6d) and satisfy the regularity constraints in (4b), the dependent variables defined by the

$$
\begin{aligned}
\sum_{m=0}^{L_{\beta}-1-\left\lceil\frac{k_{0}}{2}\right\rceil} \beta_{m} \frac{\left(2\left(L_{\beta}-m\right)-2\right) !}{\left(2\left(L_{\beta}-m\right)-2-k_{0}\right) !} & =\frac{\left(2\left(L_{\beta}-N\right)-1\right) !}{\left(2\left(L_{\beta}-N\right)-1-k_{0}\right) !} \\
\sum_{r=0}^{k_{1}}\left(\sum_{m=0}^{L_{\alpha}-1-\left\lceil\frac{\left(k_{1}-r\right)}{2}\right\rceil} \alpha_{m} \frac{C_{r}^{k_{1}}\left(2\left(L_{\beta}-N\right)-1\right) !\left(-2 m+2 L_{\alpha}-2\right) !}{\left(2\left(L_{\beta}-N\right)-1-r\right) !\left(-2 m+2 L_{\alpha}-2-k_{1}+r\right) !}\right) & =\frac{\left(2\left(L_{\alpha}+L_{\beta}-M\right)-4\right) !}{\left(2\left(L_{\alpha}+L_{\beta}-M\right)-4-k_{1}\right) !}
\end{aligned}
$$


equality constraints (4b) are expressed as a linear combination of the independent variables as follows:

$$
\left[\begin{array}{ll}
\boldsymbol{B}_{n-r} & \boldsymbol{B}_{r}
\end{array}\right] \cdot\left[\begin{array}{c}
\boldsymbol{\beta}_{n-r} \\
\boldsymbol{\beta}_{r}
\end{array}\right]=\boldsymbol{c}_{\beta, k_{0}}
$$

where $\boldsymbol{B}=\left[\boldsymbol{B}_{n-r} \boldsymbol{B}_{r}\right], \boldsymbol{\beta}=\left[\begin{array}{c}\boldsymbol{\beta}_{n-r} \\ \boldsymbol{\beta}_{r}\end{array}\right]$ and the subscripts $r$ and $n-$ $r$ indicate the redundant and nonredundant parts, respectively. Using (7), $\boldsymbol{\beta}$ can be written in terms of $\boldsymbol{\beta}_{n-r}$ as

$$
\boldsymbol{\beta}=\left[\begin{array}{c}
0 \cdot \boldsymbol{I}_{n-r} \\
\boldsymbol{B}_{r}^{-1} \boldsymbol{c}_{\beta, k_{0}}
\end{array}\right]+\left[\begin{array}{c}
\boldsymbol{I}_{n-r} \\
-\boldsymbol{B}_{r}^{-1} \boldsymbol{B}_{n-r}
\end{array}\right] \boldsymbol{\beta}_{n-r} .
$$

Substituting (8) into (6c) and redefine $\boldsymbol{x}^{T}=\left[\begin{array}{ll}\delta & \boldsymbol{\beta}_{n-r}^{T}\end{array}\right]$, we still have the objective function and the constraints affine in $\boldsymbol{x}$. In other words, it is still a standard SDP problem. The design of $\alpha\left(e^{j \omega}\right)$ using SDP is similar to that of $\beta\left(e^{j \omega}\right)$. After the design of $\beta\left(e^{j \omega}\right)$ and hence the analysis filter $H_{0}\left(e^{j \omega}\right)$, the desired response of $\alpha_{d}\left(e^{j \omega}\right)$ is obtained from (2) as $\left(e^{j(-M-1 / 2) \omega} / H_{0}\left(e^{j \omega / 2}\right)\right)$. If $H_{0}\left(e^{j \omega}\right)$ is a good lowpass filter, then obviously, $\alpha_{d}\left(e^{j \omega}\right)$ approximates a unit delay $\left(\alpha_{d}\left(e^{j \omega}\right) \approx e^{j(-M+N-1 / 2)} \omega\right)$, which is similar to the case of $\beta\left(e^{j \omega}\right)\left(\beta_{d}\left(e^{j \omega}\right)=e^{j(-N-1 / 2) \omega}\right)$. Furthermore, the regularity condition imposed on $\alpha\left(e^{j \omega}\right)$ is a system of linear equality constraints as shown in (4). Therefore, the design of $\alpha\left(e^{j \omega}\right)$ can be done similar to that of $\beta\left(e^{j \omega}\right)$ in the form of (6) and its design is given as follows

$$
\begin{aligned}
& \min \delta \\
& \eta_{R, \alpha}^{2}\left(\omega_{k}\right)+\eta_{I, \alpha}^{2}\left(\omega_{k}\right) \leq \delta, \quad k=1, \cdots K,
\end{aligned}
$$

where $\eta_{I, \alpha}(\omega)=\boldsymbol{\alpha}^{T} \cdot \boldsymbol{s}+\boldsymbol{I} \boldsymbol{m}\left\{\alpha_{d}\left(e^{j \omega}\right)\right\}, \eta_{I, \alpha}(\omega)=\boldsymbol{\alpha}^{T} \cdot \boldsymbol{s}+$ $\operatorname{Im}\left\{\alpha_{d}\left(e^{j \omega}\right)\right\}, \boldsymbol{c}=\left[\cos (0 \cdot \omega) \cos (1 \cdot \omega) \cdots \cos \left(\left(L_{\alpha}-1\right) \cdot \omega\right)\right]$, $\boldsymbol{s}=\left[\sin (0 \cdot \omega) \sin (1 \cdot \omega) \cdots \sin \left(\left(L_{\alpha}-1\right) \cdot \omega\right)\right]$ and the frequencies points, $\omega_{k}$ 's, $k=1, \cdots K$ are the discretization of the band of interest $\omega \in\left[0,2 \omega_{\alpha p}\right]$. Using the Schur complement [8]-[10], the above design is equivalent to

$$
\begin{aligned}
& \min \delta \\
& F_{k}(\boldsymbol{h}) \geq 0, \quad k=1, \cdots K .
\end{aligned}
$$

where

$$
F_{k}(\boldsymbol{h})=\left(\begin{array}{ccc}
\delta & \eta_{R, \alpha}\left(\omega_{k}\right) & \eta_{I, \alpha}\left(\omega_{k}\right) \\
\eta_{R, \alpha}\left(\omega_{k}\right) & 1 & 0 \\
\eta_{I, \alpha}\left(\omega_{k}\right) & 0 & 1
\end{array}\right) .
$$

Since $F_{k}(\boldsymbol{h})$ is affine in $\boldsymbol{\alpha}$, it is equivalent to a set of linear matrix inequalities (LMI) [9]. Define the augmented variable $\boldsymbol{x}^{T}=\left[\begin{array}{ll}\delta & \boldsymbol{\alpha}^{T}\end{array}\right]$, it can also be cast as a standard SDP problem in (6d). Finally, it should be noted that other linear constraints other than the regularity condition can be accommodated into the proposed SDP framework without any difficulties.

\section{DESIGN EXAMPLES}

\section{A. Design Example 1}

In this example, we will design the linear-phase two-channel FIR FBs with the same specification as the one in [6] (the first example). In addition, there are four zero-order moments imposed at each of the highpass and lowpass filters. The lengths of $\beta(z)$ and $\alpha(z)$ are both equal to 8 , with $N=4$ and $M=7$. The passband edges of $H_{o}(z)$ and $H_{1}(z)$ are $0.345 \pi$ and $0.727 \pi$, respectively. The FBs are designed using the proposed SDP approach with the frequency band of interest discretized evenly

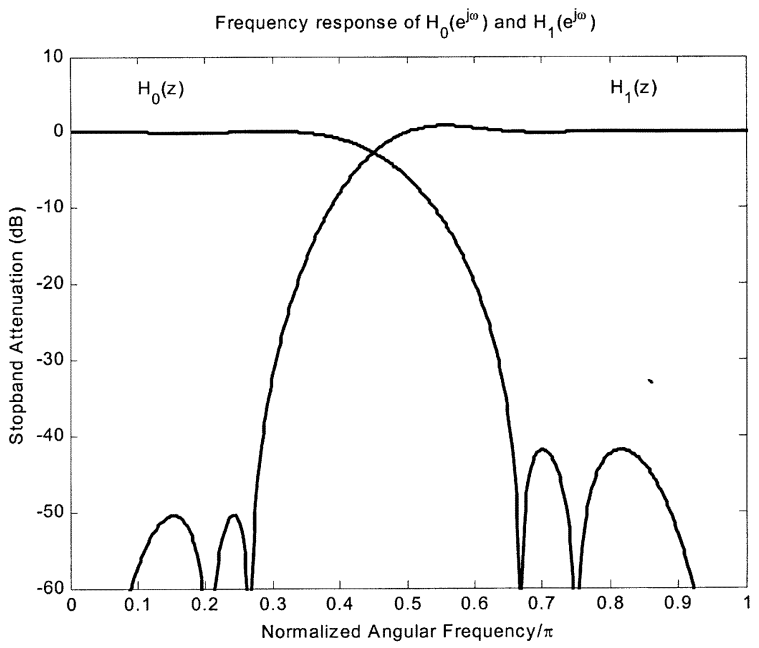

(a)

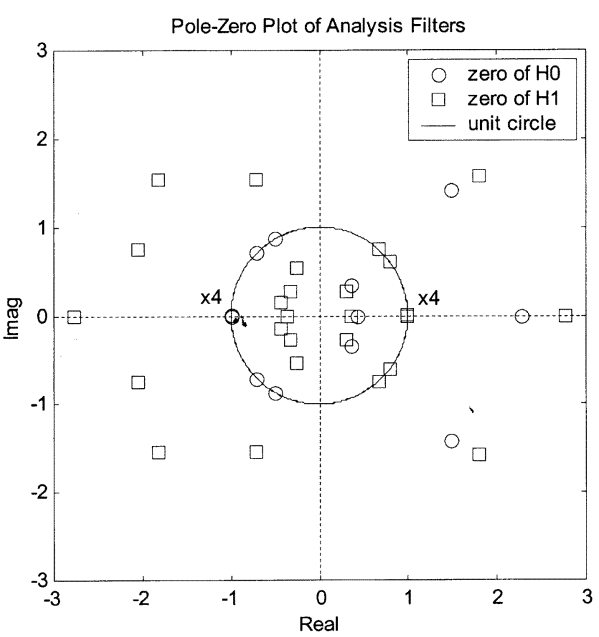

(b)

Fig. 2. (a) Frequency response and (b) zero plot of the two-channel FBs (analysis) in design example 1.

into 100 points. The frequency response, analysis scaling function and analysis wavelet function are shown in Figs. 2-4, respectively. The stopband attenuations of $H_{0}(z)$ and $H_{1}(z)$ are seen to be equiripple with values up to 40 and $50 \mathrm{~dB}$, respectively. They are higher than the one obtained by using the least squares design [6]. In fact, the stopband attenuations of $H_{0}(z)$ and $H_{1}(z)$ in the first example of [6] are around $38 \mathrm{~dB}$. Also, due to the imposed regularity, the obtained wavelet functions are very smooth.

\section{B. Design Example 2}

In this design example, another two-channel linear-phase FIR FBs with much longer filter length are designed. There are one and two zero-order moments imposed at the highpass and lowpass filters, respectively. The lengths of $\beta(z)$ and $\alpha(z)$ are 20 and 26, respectively, with $N=10$ and $M=22$. The passband edges of $H_{0}(z)$ and $H_{1}(z)$ are $0.44 \pi$ and $0.56 \pi$, respectively. The frequency band of interest is discretized evenly into 300 points, and the frequency response obtained is plotted in Fig. 5. It can be seen that the stopbands of $H_{0}(z)$ and $H_{1}(z)$ are equiripple and the maximum attenuation are 45.5 and 47 $\mathrm{dB}$, respectively. Both design examples are carried out using the 


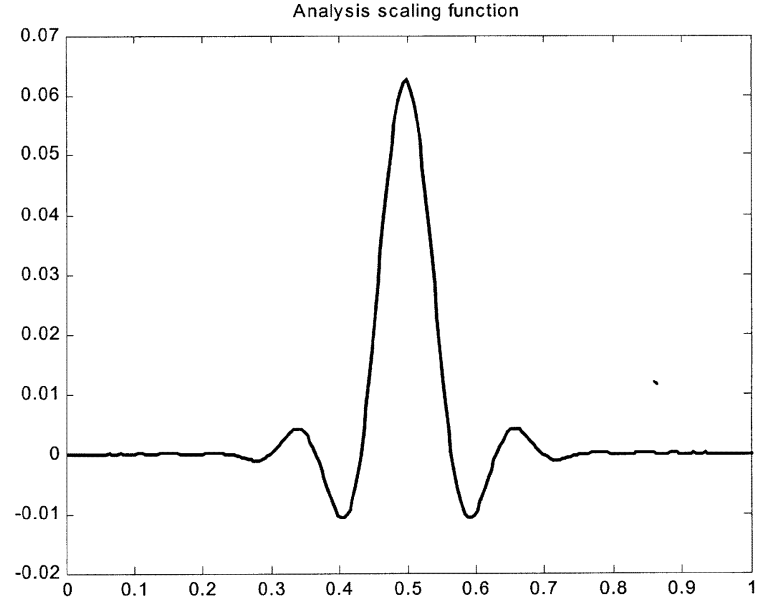

Fig. 3. Analysis scaling function of the two-channel FBs in design example 1.

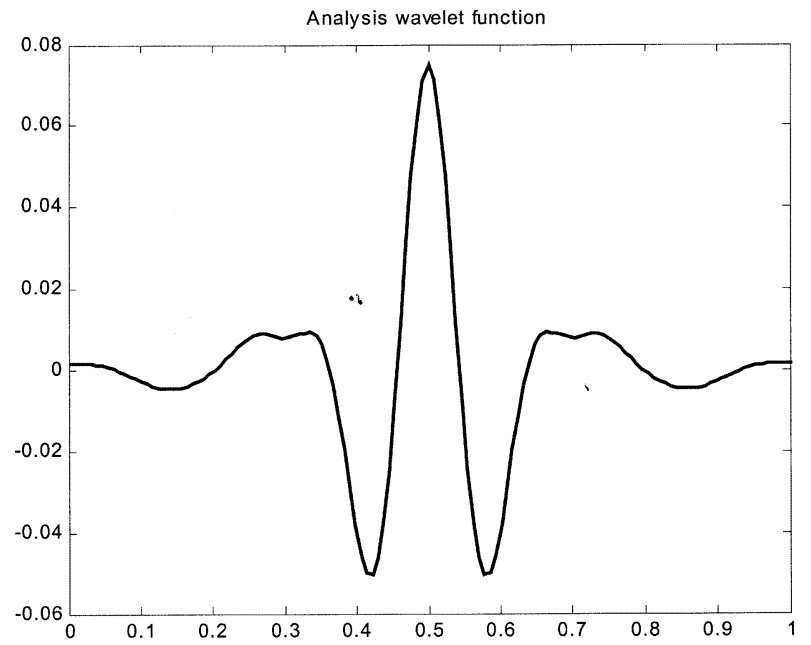

Fig. 4. Analysis wavelet function of the two-channel FBs in design Example 1.

MATLAB LMI toolbox and it takes less than 100 iterations to obtain the final results.

\section{CONCLUSION}

A new method for designing a class of two-channel PR linearphase FIR FBs and wavelets using semidefinite programming (SDP) is presented. The basic idea is to express the given $K$-regularity constraints as a set of linear equality constraints in the design variables so that the design problem using the minimax error criterion can be formulated as a SDP problem. Design examples show that the proposed method is very effective and it

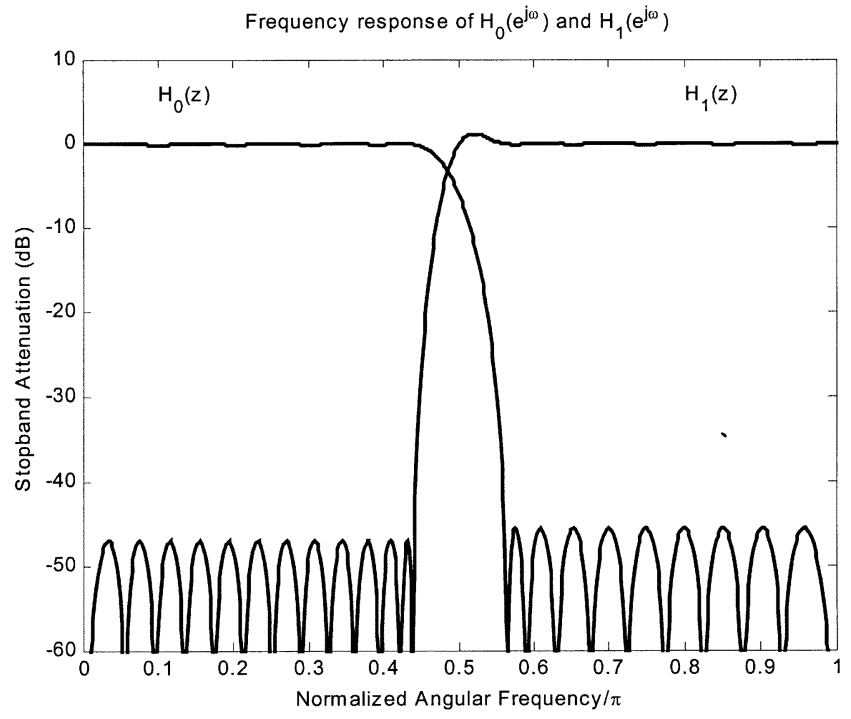

Fig. 5. Frequency response of the two-channel FBs (analysis) in design Example 2.

yields equiripple stopband response while satisfying the given $K$-regularity condition.

\section{REFERENCES}

[1] S. M. Phoong, C. W. Kim, P. P. Vaidyanathan, and R. Ansari, "A new class of two-channel biothogonal filter banks and wavelet bases," IEEE Trans. Signal Processing, vol. 43, pp. 649-664, Mar. 1995.

[2] J. S. Mao, S. C. Chan, W. Liu, and K. L. Ho, "Design and multiplier-less implementation of a class of two-channel PR FIR filterbanks and wavelets with low system delay," IEEE Trans. Signal Processing, vol. 48, pp. 3379-3394, Dec. 2000.

[3] W. Liu, S. C. Chan, and K. L. Ho, "Low-delay perfect reconstruction two-channel FIR/IIR filter banks and wavelet bases with SOPOT coefficients," in Proc. IEEE ICASSP, Istanbul, Turkey, May 2000.

[4] S. C. Chan, J. S. Mao, and K. L. Ho, "A new design method for twochannel perfect reconstruction IIR filter banks," IEEE Signal Processing Lett., vol. 7, pp. 221-223, Aug. 2000.

[5] P. Duhamel and O. Rioul, "A Remez exchange algorithm for orthonormal wavelets," IEEE Trans. Circuits Syst. II, vol. 41, pp. 550-560, Aug. 1994.

[6] D. B. H. Tay, "Two stage, least squares design of biorthogonal filter banks," in Proc. IEEE ISCAS, vol. 1, 2000, pp. 591-594.

[7] J. S. Mao, W. S. Lu, S. C. Chan, and A. Antoniou, "Design and multiplierless implementation of two-channel biorthogonal IIR filter banks with low system delay," in Proc. ISCAS, vol. 2, 2001, pp. 465-468.

[8] W. S. Lu and A. Antoniou, "Design of digital filters and filter banks by optimization: A state of the art review," in Proc. EUSIPCO, Tampere, Finland, Sep. 2000.

[9] S. Boyd, L. Ghaoui, E. Feron, V. Balakrishnan, and V. Balakrishnan, Linear Matrix Inequalities in System and Control Theory. Philadelphia, PA: SIAM, 1994.

[10] L. Vandenberghe and S. Boyd, "Semidefinite programming," SIAM J. Rev., vol. 38, pp. 49-95, Mar. 1996.

[11] Y. Nesterov and A. Nemirovskii, Interior Point Polynomial Methods in Convex Programming. Philadelphia, PA: SIAM, 1994. 